\title{
Applicability of MOS structures in monitoring catalytic properties, as exemplified for monolayer-iron-oxide-coated porous platinum films
}

Hossein Fashandi, Markus Soldemo, Jonas Weissenrieder, Mats Gothelid, Jens Eriksson, Per Eklund, Anita Lloyd Spetz and Mike Andersson

\section{Journal Article}

\section{Tweet}

N.B.: When citing this work, cite the original article.

Original Publication:

Hossein Fashandi, Markus Soldemo, Jonas Weissenrieder, Mats Gothelid, Jens Eriksson, Per Eklund, Anita Lloyd Spetz and Mike Andersson, Applicability of MOS structures in monitoring catalytic properties, as exemplified for monolayer-iron-oxide-coated porous platinum films, Journal of Catalysis, 2016. 344, pp.583-590.

http://dx.doi.org/10.1016/j.jcat.2016.10.018

Copyright: Elsevier

\section{http://www.elsevier.com/}

Postprint available at: Linköping University Electronic Press

http://urn.kb.se/resolve?urn=urn:nbn:se:liu:diva-133890

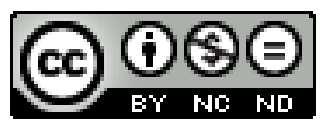




\section{Applicability of MOS structures in monitoring catalytic properties, as exemplified for monolayer-iron-oxide-coated porous platinum films}

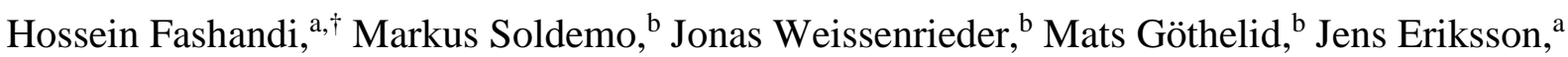
Per Eklund, ${ }^{\mathrm{a}}$ Anita Lloyd Spetz, ${ }^{\mathrm{a}}$ and Mike Andersson ${ }^{\mathrm{a}}$

a. Department of Physics, Chemistry, and Biology (IFM), Linköping University, SE-581 83 Linköping, Sweden

b. KTH Royal Institute of Technology, Material Physics, SE-164 40 Kista, Sweden † Corresponding author, hosfa@ifm.liu.se 


\section{Highlights:}

- Monolayer-iron-oxide synthesized on porous polycrystalline platinum films to serve as the metal gate in MOS devices.

- A low-temperature shift in CO oxidation characteristics for the monolayer-coated compared to non-coated polycrystalline Pt films was observed.

- An in situ characterization method for catalyst surfaces is presented based on the capacitance signal of the MOS structures.

- The MOS electrical signal, CO oxidation characteristics, and surface chemical composition remained stable for the monolayer-coated devices after $200 \mathrm{~h}$ of catalyst operation. 
Abstract:

Metal Oxide Semiconductor (MOS) capacitor devices comprised of monolayer iron oxidecoated as well as non-coated polycrystalline Pt deposited on oxidized silicon carbide substrates have been fabricated and their usefulness as realistic model systems in catalyst studies/ development evaluated. The CO oxidation characteristics of both iron oxide- and noncoated Pt catalysts were investigated using mass spectrometry, monitoring the carbon dioxide production rate for different combinations of carbon monoxide (CO) and oxygen concentrations at various temperatures. Additionally, the output capacitance of the MOS model catalysts was recorded for each individual CO oxidation activity. A low-temperature shift in CO oxidation characteristics for the monolayer-coated compared to the non-coated Pt catalysts was observed, similar to that previously reported for monolayer iron oxide grown on single-crystalline Pt substrates. A strong correlation between the output capacitance of the MOS structures and the CO oxidation characteristics was found for both monolayer- and noncoated model catalysts. Furthermore, the devices exhibit retained MOS electrical output and CO oxidation characteristics as well as an unaffected catalyst surface composition, as confirmed by photoelectron spectroscopy, even after 200 hours of continuous model catalyst operation. In addition to the implications on practical applicability of monolayer iron oxide coating on widely used polycrystalline Pt films in real-world catalysts and sensors, the findings also point to new possibilities regarding the use of MOS model systems for in-situ characterization, high-throughput screening, and tailoring of e.g. catalyst- and fuel-cellelectrode materials for specific applications.

\section{KEYWORDS:}

Monolayer iron oxide, platinum catalyst, catalytic activity, CO oxidation, field effect device, MOS capacitor, CO sensor 


\section{Introduction}

Nowadays, catalysis in all its applications is increasingly penetrating daily-life through consumer products such as enzymatically-active cleaning agents, car exhaust catalytic converters, and various gas sensors for e.g. air quality monitoring $[1,2]$. This wide and increasing range of applications has resulted in the development of a large variety of catalyst systems employing a multitude of materials designs. Tuning of catalytic properties for specific applications may, in addition to the choice of active material, be achieved through e.g. variations in nanoscale morphology $[3,4]$, the use of different support/ substrate materials [5,6], alloying with other catalytically active materials $[7,8]$ and/ or by the use of promoters [9]. Generally, an almost infinite number of possibilities exist regarding the modification of catalyst materials in order to change their activity and improve their selectivity towards different reactions. Following the near-future transition from a society largely based on the utilization of fossil raw materials into one mainly relying on renewable resources, the importance of catalytic processes is expected to increase even further in e.g. clean, resource- and cost-efficient bio-based production, power generation, and waste-water recycling $[1,10]$. Considering both this growing interest in the development of catalysis-based green technology products such as fuel cells [11] and photo-catalysts [12], and the general trend for shorter time-to-market as a means to increase overall productivity, methods for rapid and parallel study/ characterization as well as high-throughput screening of a wide variety of different catalyst formulations could thus be of broad interest.

In surface science model experiments designed for the study/ understanding of catalytic properties and characteristics, single-crystalline phases of catalytic material with well-defined crystallographic orientations are commonly used in studying the 
effect of various catalyst modifications on the catalytic properties [13-18]. In contrast, for almost all practical applications involving catalysis, the active material is comprised of well-dispersed clusters or grains, or possibly polycrystalline films, on a support material [19]. Such catalysts expose to the ambient a multitude of different crystallographic planes and defect sites such as kinks, corner sites, and vacancies, as well as the support material itself. Furthermore, standard surface science characterization methods are generally sophisticated, expensive, and exhibit limitations regarding catalyst operational conditions [20,21]. Taking this into account it would also be beneficial if cheap and rapid characterization and screening could be based on methods void of such limitations whilst still providing information on in-operando catalyst surface conditions such that e.g. trends in the gas-catalyst interaction may be assessed. Probing of the catalytic properties of surfaces in-situ and under normal operating conditions, e.g. pressure and temperature, based on fast and simple characterization methods such as standard electrical measurements could therefore be of much interest and provide further assistance in the study of catalyst modifications.

Catalytic Metal/ Oxide/ Semiconductor (MOS) structures closely resemble the structure of real-world supported metal catalysts, fuel cell electrode materials, and certain types of gas sensors [22-28]. Therefore, MOS structures could possibly serve as realistic structural platforms to screen and characterize catalytic materials, as well as to possibly gain information complementary to what is obtained from studies on single-crystalline samples for the understanding of processes on catalyst surfaces. Catalytic MOS structures form capacitors where the capacitance may be related to various catalyst surface conditions and processes such as adsorption, desorption, and reactions between adsorbed species. Simple MOS capacitance measurements may thus offer a cheap and rapid catalyst characterization method 
employing purely electrical measurements, monitoring the change in capacitance under different experimental conditions which can affect the surface catalytic reactions.

In the case of gas sensor devices based on the MOS platform [23], changes in capacitance across the MOS structure, resulting from the interaction between certain gaseous compounds and the catalytic metal film, are utilized for sensing purposes. For example, when exposing a catalytic MOS structure to hydrogen or hydrogen-containing substances, atomic hydrogen may be generated through dissociative adsorption on the catalytic metal surface. Adsorbed hydrogen atoms subsequently diffuse through the thin metal film (or in the case of a catalytic film with partial surface coverage, directly spill over to exposed patches of oxide) and adsorb on top of oxygen atoms in the surface of the oxide. The resulting hydroxide groups $(\mathrm{OH}-$ groups) [29] give rise to a change in polarization of the metal/ oxide interface, since the hydrogen is basically transferred as a proton with its mirror charge residing on the metal side of the interface. This interface polarization affects the metal-to-semiconductor electric field similarly to the application of an external positive bias on the MOS capacitor metal contact. As a result, the band bending at the surface of the semiconductor is changed and thus the width (thickness) of the depletion region (space charge region) in the near-oxide part of the semiconductor modulated. In analogy to a parallel plate capacitor for which the distance between the plates is decreased, a decrease in the depletion-region width will lead to an increase (and vice versa) in capacitance (since no mobile charges are present in the depletion region which can contribute to the capacitance, the MOS capacitance is ideally determined by the oxide- and depletion region thicknesses, their corresponding permittivity, and the metal contact area). For an n-type semiconductor, a positive bias on the metal contact induces a band bending towards the internal Fermi level and therefore a decrease in depletion region width. Thus, the capacitance increases for e.g. an n-type Pt-contact MOS capacitor when 
subjected to hydrogen. The change in capacitance reflects the change in hydrogen concentration at the interface as well as on the outer Pt surface, the capacitance output therefore being a measure of hydrogen coverage on the surface of the active catalyst. For the case of hydrogen-sensing applications, this coverage is in turn dependent on the ambient concentration of hydrogen and/or hydrogen-containing substances [30].

Pt is an outstanding catalyst that has been used as the metal gate for catalytic MOS sensor devices [2,31-33]. Pt has also been used in a wide range of other applications, e.g. as active material in car exhaust catalytic converters [34,35], in catalysts for hydrocarbon reforming in petroleum industry [19,36,37], or as electrode material in fuel cells [38]. One example of a modification by which the catalytic activity of Pt towards one or more reactions may be enhanced is the synthesis of a monolayer (ML) of metal oxide on single-crystalline $\mathrm{Pt}$ surfaces [13-18]. For ML iron oxide-modified Pt(111) single-crystal surfaces, a significant low-temperature shift of the catalytic activity to carbon monoxide (CO) oxidation, as compared to non-coated (bare) Pt crystals, have been demonstrated [13,14]. Grown on Pt(111), iron oxide forms a well-characterized FeO ML [39-41] which after exposure to mbar pressures of $\mathrm{O}_{2}$ transforms into an oxygen-rich structure, $\mathrm{FeO}_{\mathrm{x}}(1<\mathrm{x}<2)$, with no corresponding bulk phase $[13,14]$. Under oxygen-rich conditions, such a catalyst is highly active at low temperatures regarding $\mathrm{CO}$ oxidation. The reaction has been shown to proceed through the Mars-van Krevelen mechanism [14,42], as compared to the LangmuirHinshelwood mechanism of CO oxidation on non-coated Pt surfaces [19]. ML iron oxide modification of realistic supported-Pt catalysts thus presents one conceptually interesting case which may provide insights on the usefulness of the MOS model platform in characterizing and evaluating the practical performance of not only ML iron oxide-modified but also other real-world catalysts. 
ML (on average) iron oxide films have therefore been grown on poly-crystalline Pt films deposited on oxidized $\left(\mathrm{SiO}_{2}\right)$ silicon carbide $(\mathrm{SiC})$ substrates to form MOS structures and the CO oxidation characteristics of both ML iron oxide- and non-coated polycrystalline $\mathrm{Pt} / \mathrm{SiO}_{2} / \mathrm{SiC}$ catalysts obtained for various $\mathrm{CO}$ and $\mathrm{O}_{2}$ concentrations at different temperatures. The results are discussed in relation to what has previously been presented for ML iron oxide-coated and non-coated single-crystalline Pt to further evaluate the MOS models ability to bridge the gap between crystallographically well-defined well-ordered model platforms and real-world catalysts. Furthermore, the capacitance signals of both MLcoated and non-coated MOS devices are compared to the corresponding CO oxidation characteristics for different $\mathrm{CO} / \mathrm{O}_{2}$ concentration ratios and at various temperatures. A practical correlation is found between the CO oxidation characteristics and the electric output of the MOS model devices from which the applicability of capacitance measurements as a simple real-world catalyst characterization technique is discussed. The validity of the correlation upon catalyst aging, as evaluated from long-term stability studies of ML iron oxide-coated supported Pt catalysts, is reported and the implications of these findings on e.g. the applicability of MOS model systems in the study of catalyst aging briefly treated.

\section{Experimental}

\subsection{MOS structure fabrication}

The MOS capacitor structures used for materials characterization and gas exposure experiments were fabricated from 4" n-type (doping concentration $~ 3 \times 10^{18} \mathrm{~cm}^{-3}$ ) $4 \mathrm{H}-\mathrm{SiC}$ substrates. On this substrate, an n-type (doping concentration $\sim 6 \times 10^{15} \mathrm{~cm}^{-3}$ ) epitaxial layer of $2 \mu \mathrm{m}$ thickness was grown followed by thermal oxidation at $1150{ }^{\circ} \mathrm{C}$ in the presence of 
nitrous oxide $\left(\mathrm{N}_{2} \mathrm{O}\right)$, forming a $50 \mathrm{~nm}$ thick $\mathrm{SiO}_{2}$ film. Subsequently a $25 \mathrm{~nm}$ thick silicon nitride $\left(\mathrm{Si}_{3} \mathrm{~N}_{4}\right)$ film was formed through low-pressure chemical vapor deposition and densified through wet oxidation at $900{ }^{\circ} \mathrm{C}$, also creating an approximately $5 \mathrm{~nm}$ thick silicon oxide on the surface, all resulting in a dielectric stack comprising of $50 \mathrm{~nm} \mathrm{SiO} / 25 \mathrm{~nm}$ $\mathrm{Si}_{3} \mathrm{~N}_{4} / \sim 5 \mathrm{~nm} \mathrm{SiO}{ }_{2}$.

An ohmic contact was formed to the backside of the substrate through removal of the thermally grown oxide and the subsequent deposition (thermal evaporation) and rapid thermal annealing $\left(950^{\circ} \mathrm{C}\right.$, Ar atmosphere, $5 \mathrm{~min}$ ) of a $50 \mathrm{~nm}$ Ni layer (resulting in an ohmic $\mathrm{NiSi}_{\mathrm{x}} /$ SiC interface). Ring shaped metal contacts/bonding pads of $4.5 \mathrm{~mm}$ diameter were fabricated on top of the oxide stack on the front of the wafer by DC magnetron sputter deposition of 10 $\mathrm{nm} \mathrm{Ti} / 400 \mathrm{~nm}$ Pt at room temperature and an Ar pressure of $2.6 \times 10^{-3}$ mbar. The patterning for metal contact deposition was made through standard photolithographic techniques and the lift-off procedure used for defining the contacts. The Ti acts as an adhesion layer for the Pt film. All targets used were of 99.99\% purity or better. An inhomogeneous, polycrystalline Pt "layer" was deposited by DC magnetron sputtering at an Ar pressure of $6.6 \times 10^{-2}$ mbar to a total thickness of $25 \mathrm{~nm}$, covering the area on and inside the ring shaped metal contact (see Fig. 1 (a)). The elevated Ar pressure during deposition promotes the generation of a film with increased surface roughness with partial surface coverage which leaves distributed exposed substrate areas. Scanning electron microscopy (SEM) was performed in a LEO 1550 microscope for surface imaging. The surface morphology was further studied with atomic force microscopy (AFM) using a Veeco DI Dimension 3100 scanning probe microscope, equipped with the Nanoscope IV electronics, at room temperature and in lab ambient. The measurements were performed in tapping mode using Si tips (PPP-NCHR-50 from Nanosensors) with a tip radius of curvature $<7 \mathrm{~nm}$. X-ray diffraction (XRD) analysis was 
done using a Philips PW 1820 instrument $(\mathrm{Cu}(\mathrm{K} \alpha), \theta-2 \theta$ scan, aligned with the substrate (0001) peak).

\subsection{Iron oxide ML synthesis}

As-processed MOS substrates were introduced into an ultra-high vacuum chamber (base pressure at $1 \times 10^{-10}$ mbar) comprising low energy electron diffraction (LEED) optics and conventional surface science sample preparation equipment. The Pt film on the surface of the sensor was cleaned by gentle Ar sputtering at $1 \mathrm{kV}$ for $60 \mathrm{~s}$ followed by annealing to $600{ }^{\circ} \mathrm{C}$. Prior to iron deposition a saturated layer of oxygen was deposited onto the Pt surface by two cycles of annealing in $\mathrm{O}_{2}$ at $1 \times 10^{-6}$ mbar to $600{ }^{\circ} \mathrm{C}$ followed by cooling down to room temperature under the same $\mathrm{O}_{2}$ pressure. The oxygen at the surface prevents the deposited iron from alloying with the Pt and diffusing into the Pt thin film electrode. Subsequently iron was deposited from an iron rod (Goodfellow, purity $>99.9 \%$ ), mounted in an electron beam assisted evaporator (Focus EFM3, Omicron), at $10 \mathrm{nA}$ beam flux for $100 \mathrm{~s}$. The iron deposition rate was calibrated by both a quartz crystal microbalance and by deposition onto a $\mathrm{Pt}(111)$ single crystal where an ordered $\mathrm{FeO}$ structure is formed at one $\mathrm{ML}$ and was characterized by LEED $[14,39]$. After the deposition of iron, the samples were annealed in $\mathrm{O}_{2}$ gas at $1 \times 10^{-6}$ mbar to $600{ }^{\circ} \mathrm{C}$ followed by cooling down in the same ambient.

\subsection{CO oxidation characterization}

The as-processed wafer samples were diced into chips of $\sim 5 \times 5 \mathrm{~mm}^{2}$ size, each containing one MOS device, and the individual chips along with a Pt-100 temperature sensor (Heraeus Sensor-Nite GmbH) attached to a heater substrate (Heraeus Sensor-Nite GmbH, ReinhardHeraeus-Ring, Kleinostheim, Germany) by means of a high temperature, non-conducting 
ceramic die. The heater substrates were in turn mounted on 16-pin TO8 headers and together with the Pt-100 temperature sensors electrically connected by spot welding to two pairs of pins on the TO8 header. Electrical connections to the MOS capacitor devices were made by gold wire bonding to the pins of the TO8 header. This arrangement (see also Fig. 1(a)) was used for all electrical and gas characterizations of the MOS devices.

For studying the effect of the ML iron oxide modification on the CO oxidation characteristics of silica supported Pt catalysts, MOS samples with and without the ML iron oxide synthesized on the surface, with all other features and processing-details being identical, were mounted in a test cell connected to a gas-feed line and exposed to $500 \mathrm{ppm} \mathrm{CO}$ in $5 \% \mathrm{O}_{2}$, with $\mathrm{N}_{2}$ as the carrier gas at different temperatures ranging from 100 to $240{ }^{\circ} \mathrm{C}$ (in steps of 20 ${ }^{\circ} \mathrm{C}$ or less). The purity of all gases used was of $99.99 \%$ or better. The gas flow was maintained at $100 \mathrm{ml} / \mathrm{min}$ using standard mass flow controllers (Bronkhorst High-Tec B.V., Nijverheidsstraat 1A, NL-7261 AK Ruurlo, The Netherlands) while the carbon dioxide $\left(\mathrm{CO}_{2}\right)$ production was monitored through a Hiden analytical HPR20 mass spectrometer connected to the gas-feed line downstream of the test cell.

In order to compare the CO oxidation properties between the iron oxide coated and the noncoated MOS samples, they were exposed to CO concentrations ranging from 100 to 900 ppm in 3,5 , and $7 \%$ of $\mathrm{O}_{2}$ in $\mathrm{N}_{2}$, with the latter as the carrier gas, with $100 \mathrm{ml} / \mathrm{min}$ of overall flowrate. The MOS capacitance output (capacitance across the MOS structure) was recorded using a Boonton 7200 capacitance meter (Boonton Electronics, Parsippany, NJ, USA). Each CO pulse was maintained for $120 \mathrm{~s}$ and separated by $240 \mathrm{~s}$ intervals without CO exposure but with the same concentration of $\mathrm{O}_{2}$. The measurements were carried out at different 
temperatures ranging from $145{ }^{\circ} \mathrm{C}$ to $220{ }^{\circ} \mathrm{C}$. The long-term stability of the ML-coated samples was studied by continuously operating the MOS catalyst samples at $190{ }^{\circ} \mathrm{C}$ and exposing the structures to CO pulses from 400 ppm to 700 ppm (in steps of $100 \mathrm{ppm}$ ) for 120 $\mathrm{s} / 120 \mathrm{~s}$ of exposure/ recovery in $5 \% \mathrm{O}_{2}$ in $\mathrm{N}_{2}$. The long-term stability experiments were continued for 200 hours.

\subsection{Photoelectron spectroscopy}

Samples with and without ML iron oxide coating were investigated at the photoelectron spectroscopy (PES) branch of beamline I311 at Maxlab, Sweden. I311 is an undulator based beamline and the end station was equipped with a Scienta SES200 electron analyzer, LEED, and standard surface science preparation equipment [43]. The Fe 2p-region was measured using photon energy of $1100 \mathrm{eV}$ and the $\mathrm{O}$ 1s-region using a photon energy of $900 \mathrm{eV}$. All spectra are normalized on the low binding energy side and their energy scales are corrected to the Fermi edge of the samples.

\section{Results and discussion}

\subsection{CO oxidation characteristics}

The overall MOS design applying to the ML iron oxide as well as non-coated Pt catalysts used in this study is shown in Fig. 1 (a). Fig. 1 (b) displays a SEM image of the Pt film which acts as both active catalyst material and metal electrode in the MOS capacitors. The difference in the brightness of Pt compared to the underlying $\mathrm{SiO}_{2}$ shows the partial surface-coverage of the Pt film. Fig. 1 (c) is an AFM image of the surface morphology of Pt. The surface is, in contrast to its single-crystal counterpart, comprised of densely distributed valleys and peaks. 
Fig. 1 (d) is an X-ray diffractogram of the MOS sample showing diffraction peaks of Pt from different orientations. This result shows that the Pt film is polycrystalline with a degree of preferred (111) orientation.

Fig. 2 presents mass spectrometry data collected downstream of the MOS catalysts during their exposure to a continuous flow of $500 \mathrm{ppm} \mathrm{CO}$ in a background of $5 \% \mathrm{O}_{2}$ in $\mathrm{N}_{2}$ over a range of different temperatures. As can be seen, the onset of increased $\mathrm{CO}_{2}$ production with increasing temperature occurs at about $40{ }^{\circ} \mathrm{C}$ lower temperature for the $\mathrm{ML}$ iron oxide-coated samples compared to the non-coated ones. A similar difference in CO oxidation characteristics between ML iron oxide- and non-coated Pt has earlier been found using singlecrystal Pt $[14,15]$. In both the previous and current cases, this behavior corresponds to a higher low-temperature catalytic activity of ML-coated as compared to non-coated Pt for the CO oxidation process.

These findings thus point in the direction of the earlier reported low-temperature shift in CO oxidation activity being retained also for the more realistic MOS model catalyst, enabling the evaluation of its possible use for bridging the gap between single-crystal and real-worldcatalyst studies. In addition, as seen from Fig. 2 the amount of produced $\mathrm{CO}_{2}$ per unit time seems higher for the ML-coated samples compared to the non-coated ones. This observation may be indicative of an overall higher $\mathrm{CO}_{2}$ production rate for the $\mathrm{ML}$ iron oxide- compared to the non-coated samples which has also been reported for the case of ML iron oxide-coated single-crystal Pt [14]. To be able to make a firm statement on this, any difference in active catalyst surface area between the different samples first needs to be evaluated. This, however, 
is difficult with available techniques such as the Brunauer-Emmett-Teller (BET) due to a comparably small surface area of the samples, on the order of $10-20 \mathrm{~mm}^{2}$.

\subsection{MOS model system characteristics}

Fig. 3 displays the output capacitance for both non-coated and ML-coated MOS capacitors. The responses are acquired during consecutive two-minute exposures to $\mathrm{CO}$ in a background of $\mathrm{O}_{2}$ and $\mathrm{N}_{2}$, interposed by four-minute exposures to just $\mathrm{O}_{2}$ and $\mathrm{N}_{2}$ (maintaining a constant gas flow of $100 \mathrm{ml} / \mathrm{min}$ over the MOS structures throughout the measurement sequence). The CO concentrations of consecutive exposures ranged from 100 to $900 \mathrm{ppm}$ and the measurements were conducted for different temperatures in the range of 145 to $220{ }^{\circ} \mathrm{C}$. For each temperature, the $\mathrm{CO}$ exposures were repeated for different background $\mathrm{O}_{2}$ concentrations; 3, 5, and 7\%. As can be seen from Fig. 3, the capacitance generally increases upon $\mathrm{CO}$ exposure while recovering to its baseline value when $\mathrm{CO}$ is removed from the gasfeed line. In large, the results also show that the capacitance signal exhibits a switch between a "high" and a "low" response ("large” / "small” change in capacitance) when keeping the CO exposure level constant and increasing either the $\mathrm{O}_{2}$ concentration or the temperature. The former case may be exemplified by the change in response for the ML-coated MOS samples operated at $145{ }^{\circ} \mathrm{C}$ when increasing the $\mathrm{O}_{2}$ concentration from 3 to $5 \%$, whereas the latter is exemplified by the change in response for the same MOS samples when increasing the temperature from 145 to $160{ }^{\circ} \mathrm{C}$ under constant exposure to $500 \mathrm{ppm} \mathrm{CO}$ and $5 \% \mathrm{O}_{2}$ (see highlighted exposures in Fig. 3). Disregarding irregularities caused by memory effects and/ or the short recovery time between exposures, the one overall trend regarding MOS capacitor output valid for both ML iron oxide- and non-coated Pt catalysts is the larger $\mathrm{CO} / \mathrm{O}_{2}$ ratio required to generate a "high" response when the temperature is increased. 
In addition to the switch being more clear and distinct in the case of ML-coated as compared to non-coated Pt samples, when comparing corresponding $\mathrm{CO}$ and $\mathrm{O}_{2}$ concentrations, this switch is also seen to occur at lower temperatures for the ML iron oxide-modified MOS capacitors compared to the non-coated ones. The less distinct difference between what can be regarded as low and high responses for the non-coated samples at lower temperatures (at least when compared to the ML-coated ones) might be explained by the longer time needed in order to reach steady-state conditions. As an example, consider the changes in capacitance of the non-coated samples upon exposure to $100 \mathrm{ppm} \mathrm{CO}$ at $160{ }^{\circ} \mathrm{C}$ and 3 and $5 \% \mathrm{O}_{2}$, respectively. At a first glance, it might be hard to judge if the response at $3 \% \mathrm{O}_{2}$ should be considered "low" or "high". However, when taking into account that steady-state conditions have not been reached for the 3\% case (the signal is still increasing at the end of the $100 \mathrm{ppm}$ CO pulse), whereas such conditions prevail for the $5 \%$ case (i.e. the signal remains at a constant magnitude during the latter part of the CO exposure), it seems clear that the response (the output capacitance signal) at $3 \% \mathrm{O}_{2}$ could be considered as "high". Only above $200{ }^{\circ} \mathrm{C}$ does the response of the non-coated MOS samples reach true steady-state conditions soon after being exposed to CO. For the ML-coated samples, however, the signals reach steadystate conditions more readily also at lower temperatures. The faster attainment of steady-state conditions for the ML-coated MOS capacitors as compared to the non-coated ones below 200 ${ }^{\circ} \mathrm{C}$ could also be a further indication of the higher catalytic activity discussed in relation to the results presented in Fig. 2. Additionally, both the attainment of steady-state and the initial rates of change in MOS capacitance signal are generally dependent on reaction conditions. Generally speaking, the higher the reaction temperature, the higher the initial rate of capacitance change and the faster attainment of steady-state conditions. Furthermore, in addition to the much smaller change in capacitance observed at the higher $\mathrm{CO}: \mathrm{O}_{2}$ ratios, the 
recorded data also seem to suggest that the time to reach steady-state conditions are shorter for the lower $\mathrm{CO}: \mathrm{O}_{2}$ ratios.

The results for the non-coated samples are in accordance with earlier findings and can briefly be explained as follows: in an oxygen-containing atmosphere and in the absence of $\mathrm{CO}$, the active catalytic surface of the non-coated Pt film is assumed to be basically covered by oxygen atoms, depending on the ambient temperature accompanied by the transfer of more or less of negative charge from Pt to the chemisorbed oxygen. The higher the temperature the more charge transferred to the adsorbed oxygen species. Adsorbed oxygen anions may also spill over between the Pt metal and oxide support surfaces. In the presence of CO molecules and at an appropriate temperature [44,45], $\mathrm{CO}$ adsorbing on the Pt surface is oxidized to $\mathrm{CO}_{2}$ by surface-adsorbed oxygen. The consumption of previously adsorbed oxygen atoms/ ions on the active area leaves free adsorption sites which can be occupied by either CO molecules or other oxygen atoms/ ions. The lower the temperature and the higher the $\mathrm{CO} / \mathrm{O}_{2}$ ratio of the ambient atmosphere, the more likely it is that a CO molecule, rather than an oxygen atom, will be adsorbed on the metal surface and occupy a free adsorption site. For constant CO and $\mathrm{O}_{2}$ concentrations, the Pt surface will thus revert to being almost depleted of oxygen when decreasing the temperature below the one for which the probability is higher that CO molecules rather than oxygen will occupy free adsorption sites. The CO oxidation reaction has become self-poisoned and the $\mathrm{CO}_{2}$ production rate significantly decreased.

Reduced coverage of adsorbed oxygen anions on the oxide surface, following the reduction in spill-over of oxygen from Pt to the oxide surface, leads, in itself, to a change in the catalytic metal-to-semiconductor substrate electric field and therefore directly contributes to the 
observed change in capacitance upon CO exposure for both kinds of samples [22]. Additionally, for an oxygen-depleted outer Pt surface, the rate of oxidation of adsorbed hydrogen (originating from the natural occurrence of a low level of hydrogen and water existing in any gas mixture) and its subsequent desorption (in the form of water) is reduced. A larger number of hydrogen atoms may thus diffuse through the Pt film or spill over from the Pt to the oxide and adsorb to form $\mathrm{OH}$ groups on the oxide surface. As discussed in the introduction part, this also contributes to the modulation of the metal-to-semiconductor electric field and thus the capacitance across the MOS device $[27,45]$.

The difference in electrical output characteristics between the two different kinds of samples might be explained according to the mechanism discussed above, taking into account the lowtemperature shift in CO oxidation activity observed for the ML iron oxide- as compared to the non-coated polycrystalline Pt catalyst (see Fig. 2). As concluded from Fig. 3 and already briefly noted above, the switch between a high and a low response of the MOS capacitors upon exposure to one particular combination of $\mathrm{CO}$ and $\mathrm{O}_{2}$ concentrations occurs at a lower temperature for the ML iron oxide-coated MOS samples than for the non-coated ones. This pattern applies generally, irrespective of the $\mathrm{CO} / \mathrm{O}_{2}$ combination studied. Moreover, when comparing the results obtained for ML- and non-coated MOS capacitor samples, the average down-shift of temperature at which the switching occurs is approximately $40-50{ }^{\circ} \mathrm{C}$, which corresponds well with the low-temperature shift in CO oxidation characteristics found for ML-iron oxide modified compared to non-coated Pt catalysts.

Furthermore, from Fig. 3 it can be seen that the ML-coated samples exhibit no visible change in capacitance upon exposure to any CO concentration at temperatures above $200{ }^{\circ} \mathrm{C}$. In contrast, at least small responses may be noticed for all CO exposures and at all temperatures in the case of non-coated Pt catalyst samples. This latter observation is also in accordance 
with findings from earlier studies, for which small changes in capacitance upon exposure to CO in the range of $100-900 \mathrm{ppm}$ have been measured even up to $400{ }^{\circ} \mathrm{C}$ [44]. Assuming for the moment that the generally higher catalytic activity of the ML iron oxide- as compared to non-coated Pt discussed in relation to the results displayed in Fig. 2 would be valid, this difference could find its explanation in the more efficient Mars-van Krevelen CO oxidation mechanism of the ML-coated as compared to the non-coated Pt catalyst, especially at higher temperatures. For the assumed Mars-van Krevelen mechanism, the adsorbing CO reacts with the iron oxide bound (the crystal bound) oxygen in forming $\mathrm{CO}_{2}$, which subsequently desorbs from the surface. So created oxygen vacancies are re-occupied mainly through dissociative adsorption of molecular oxygen from the gas-phase [14]. The high catalytic activity of ML iron oxide-coated as compared to non-coated samples generally reduces the residence time, and thereby the coverage of $\mathrm{CO}$ molecules on the surface (considering the same $\mathrm{CO}$ concentrations for both kinds of samples). Furthermore, with the CO oxidation mainly taking place through reduction of the iron oxide and the created oxygen vacancies basically being reoccupied through the interaction with gas-phase oxygen, the processes of spill-over and reverse spill-over may in this case be non-existent or negligible. As a consequence, the absence of or at least an almost constant coverage of oxygen anions on the oxide surface might thereby be maintained even upon addition of CO, within certain limits, to the gas-feed. Furthermore, it has previously been suggested that hydrogen from the ambient do not form any hydroxyl groups on the oxygen-rich surface of ML iron oxide-coated Pt structures [14]. The rapid oxidation and removal of adsorbed hydrogen atoms is, on the other hand, a reaction which for non-coated Pt-based catalysts becomes more efficient at temperatures above 200 ${ }^{\circ} \mathrm{C}$. As a result, any possible changes in the oxygen anion coverage and hydrogen adsorption at the $\mathrm{Pt} / \mathrm{SiO}_{2}$ interface can be regarded as negligible and as such consistent with the observed lack of even small changes in MOS capacitance upon CO exposure at temperatures above 200 
${ }^{\circ} \mathrm{C}$ for the ML iron oxide coated capacitors. Conversely, the somewhat lower CO oxidation activity, and thus less efficient removal of CO from the surface, leaves a slightly higher surface CO coverage for the non-coated MOS catalysts as compared to the ML-coated ones. Thus, the supported non-coated MOS samples may exhibit a small but significant reduction in coverage of adsorbed oxygen atoms and anions upon CO exposure. Together with a possible slight reduction in hydrogen consumption (as a result of the small decrease in surface oxygen coverage) and corresponding increase of adsorbed hydrogen at the $\mathrm{Pt} / \mathrm{SiO}_{2}$ interface, this could result in a detectable change of the MOS capacitance. If assumed correct, the absence of hydroxyl group formation on the ML iron oxide coated Pt samples could also provide one explanation to the generally observed smaller changes in MOS capacitance for the ML compared to the non-coated samples.

There are thus strong indications of a correlation between the $\mathrm{CO}$ oxidation reaction conditions on the surface of the active catalyst material(s) and the MOS capacitance signal. These indications support the possible use of MOS-based model systems in characterizing and understanding as well as in the tuning and tailoring of CO oxidation (and possibly other) catalyst materials for specific applications. Especially in respect of the promising possibilities to relate inexpensive measurements of simple electrical parameters, such as rate of capacitance change, to reactivity under many different conditions and for a large variety of catalyst formulations, the method could be of interest for high-throughput catalyst screening. Of particular importance for the development of fuel cell electrode and sensors, both of which may be regarded as almost 2D structures, the MOS model system also relaxes demands on large surface area samples and on very sensitive and/ or expensive surface analysis equipment. In using the MOS model structures additional information regarding the conditions on the surface or at certain interfaces of the catalyst materials may also be 
provided, e.g. the presence of hydrogen at the active phase of the catalyst, which might otherwise be complicated to obtain. Furthermore, this model system resembles a real catalyst and can be used under realistic conditions, such as elevated temperatures and high pressures. With the possibility for multiplexing of electrical signals many small-sized MOS samples of the same or different catalyst materials can also be investigated under different conditions simultaneously, increasing the throughput and shortening screening times, using inexpensive equipment.

The confirmed low-temperature shift of the CO oxidation and CO response characteristics of realistic, practically usable ML iron oxide coated supported polycrystalline Pt films may also offer new possibilities for modification of Pt-based catalysts and sensors. These could include the possibility to tune e.g. the practical operation temperature (lower temperature for low power consumption), conversion efficiency, turnover number, and selectivity of both catalysts and sensors as well as the response time, sensitivity and/or dynamic range of gas sensors. For example, the higher catalytic activity towards CO oxidation of the ML-coated samples compared to the non-coated ones provides a route towards fabrication of a gas sensor with the ability to monitor one or more other substances without CO cross-sensitivity issues at temperatures from $220{ }^{\circ} \mathrm{C}$, even for fairly large CO concentrations. One practical application for which this would be of interest is found in the monitoring of ammonia slip from power plants employing selective reduction of flue gas nitrogen oxides through the addition of ammonia to the CO containing flue gas [30].

It should finally be emphasized that in order to exclude any possible effect of pretreatment steps prior to iron deposition on the characteristics of the ML-coated MOS samples, all 
synthesis steps for the growth of iron oxide were carried out also for non- coated MOS samples, excluding the evaporation of iron. Following the processing, all gas exposures were carried out in exactly the same manner and with the same pre-treatments as detailed in the methods section. The obtained experimental results have not revealed any significant difference in characteristics between the non-coated Pt MOS samples which have gone through the iron oxide pre- and post-treatments and ordinary non-coated Pt sensors [25]. These results rule out any significant contribution from pre- or post-treatment steps of the iron oxide synthesis process on the characteristics of the ML-iron-oxide-coated samples as compared to non-coated ones.

\subsection{Long-term stability}

The stability of the ML iron oxide coated Pt catalyst has also been studied over 200 hours of operation (continuously repeated $120 / 120 \mathrm{~s}$ of CO exposure/ recovery) at $190{ }^{\circ} \mathrm{C}$. Any differences between the initial and final capacitance-signal characteristics for the different CO concentrations was thereby evaluated. In Fig. 4 the capacitance-signal characteristics towards one particular set of conditions (the consecutive exposure to $400-700 \mathrm{ppm}$ of $\mathrm{CO}$ in $5 \% \mathrm{O}_{2}$ ) is compared for the measurement sequences corresponding to the start and the end of the 200 hours operation. As can be seen, the characteristics remain essentially unchanged over the 200 hours of operation. As a complement, the state of the ML iron oxide after 50 and 200 hours of operation was studied through photoelectron spectroscopy (PES) measurements on two iron oxide coated MOS samples and any changes in the chemical bonding of the iron oxide evaluated. The corresponding results are shown in Fig. 5. The Fe 2p core level spectra from samples exposed in the reaction chamber confirm that the iron oxide film is still present at the surface even after extended exposures (200 h). Moreover, the line profile and binding energy of $\mathrm{Fe} 2 \mathrm{p}$ also show that the iron is in oxidized form (the binding energy of $\mathrm{Fe} 2 \mathrm{p}_{3 / 2}$ is 
approximately $710.0 \mathrm{eV}$ for the $50 \mathrm{~h}$ sample and $710.8 \mathrm{eV}$ for the $200 \mathrm{~h}$ sample, see inset in Figure 5). The chemical composition is likely to be $\mathrm{FeO}$ or $\mathrm{Fe}_{3} \mathrm{O}_{4}$, but the exact stoichiometry is difficult to determine for this complex material system. No discernable satellites were observed. For $\mathrm{Fe}_{2} \mathrm{O}_{3}$, a satellite has been reported at around $719.3 \mathrm{eV}$ [46]. The spectra in Fig. 5 show no sign of this satellite, indicating that the film is not of $\mathrm{Fe}_{2} \mathrm{O}_{3}$ composition. The $\mathrm{O} 1 \mathrm{~s}$ spectra show no peak shift between the two investigated iron-oxide-coated samples (binding energy $532.6 \mathrm{eV})$.

However, the $\mathrm{O} 1 \mathrm{~s}$ peak for the sample without an iron oxide layer is shifted $0.4 \mathrm{eV}$ towards higher binding energies (533.0 eV) compared to the $\mathrm{O}$ 1s peaks for the samples with the iron oxide film. A significant part of the $\mathrm{O}$ 1s signal is interpreted to originate from oxygen in the $\mathrm{SiO}_{2}$ under the partially-covering Pt film [47]. The shift towards lower binding energy for the ML iron oxide coated samples stems from the oxygen in iron oxide [46]. In summary, no significant changes to the ML iron oxide could be observed on this time scale.

The results of the long-term stability measurements indicate that the above-reported CO oxidation characteristics are repeatable over time and that no changes, neither with respect to signal output characteristics nor to elemental composition or chemical binding, have occurred over the course of the experiments. Furthermore, the long-term stability measurements indicate that ML-iron-oxide modification could be feasible also for the case of real-world supported catalysts as well as for sensor devices. Both these applications require stability over prolonged periods of time. 


\section{Conclusions}

In comparing the CO oxidation characteristics of a ML iron oxide coated (on average) to that of a non-coated supported polycrystalline Pt catalyst, the existence of a similar lowtemperature shift in CO oxidation activity has been confirmed, as previously reported for ML iron oxide modified single-crystalline Pt. The ML-coated Pt has also been shown to exhibit stable characteristics over more than 200 hours of operation, both in respect of chemical composition and its interaction with CO. In addition to the practical use of ML metal oxide modified Pt catalysts in real applications such as low-temperature CO oxidation catalysis and CO sensors, this is also promising for the use of MOS model systems for bridging the gap between single-crystal and real-world catalysts.

Furthermore, in fabricating both the ML- and the non-coated Pt catalysts as MOS capacitor structures, the output capacitance could be compared to the CO oxidation characteristics of both catalysts when subjected to different $\mathrm{CO}$ and $\mathrm{O}_{2}$ concentrations at different temperatures. The results strongly indicate a correlation between the momentary surface conditions during exposure to different $\mathrm{CO}$ and $\mathrm{O}_{2}$ mixtures and the electrical output from the MOS-capacitorbased model catalyst system. This correlation is present for both ML iron oxide-coated and non-coated Pt MOS samples and, if proven general in nature, opens possibilities to follow the state of the CO oxidation and possibly any other reaction electronically, without the need to explicitly monitor the actual surface coverage of e.g. $\mathrm{CO}$ or the $\mathrm{CO}_{2}$ production. Additionally, some results from the measurements indicate an overall higher catalytic activity (in terms of catalytic turnover), resulting in higher $\mathrm{CO}_{2}$ production per unit of time from the ML iron oxide coated sample as compared to the non-coated Pt catalyst, similar to what has been reported based on ML iron oxide-coated single-crystal Pt. Furthermore, the obtained results may also indicate a relation between a higher catalytic activity and the rate of change in 
capacitance output. This could prove useful for future work on studying and understanding catalyst materials and combinations of materials. Overall, these MOS characteristics might offer new possibilities for screening the performance of various catalyst materials, fuel cell electrodes and/or gas sensors, for example in terms of activity, conversion efficiency, selectivity, response time, sensitivity, and dynamic range.

Acknowledgements

We gratefully acknowledge the support from the Maxlab staff and from the VINN Excellence Center in research and innovation on Functional Nanoscale Materials (FunMat) by the Swedish Governmental Agency for Innovation Systems (VINNOVA) as well as the Swedish Research Council (VR) for their financial support. P. E. also acknowledges support from the Swedish Foundation for Strategic Research (SSF) through the Future Research Leaders 5 program. 


\section{References:}

[1] S.L. Suib, New and Future Developments in Catalysis: Catalysis for Remediation and Environmental Concerns, Elsevier Science, 2013.

[2] G. Korotcenkov, Handbook of Gas Sensor Materials: Properties, Advantages and Shortcomings for Applications Volume 1: Conventional Approaches, Springer New York, 2013.

[3] A. Chen, P. Holt-Hindle, Platinum-based nanostructured materials: synthesis, properties, and applications., Chem. Rev. 110 (2010) 3767-804.

[4] F. Yang, D. Deng, X. Pan, Q. Fu, X. Bao, Understanding nano effects in catalysis, Natl. Sci. Rev. 2 (2015) 183-201.

[5] M. Inaba, Y. Kintaichi, H. Hamada, Cooperative effect of platinum and alumina for the selective reduction of nitrogen monoxide with propane, Catal. Letters. 36 (1996) 223227.

[6] E. Yoo, T. Okada, T. Kizuka, J. Nakamura, Effect of carbon substrate materials as a Pt-Ru catalyst support on the performance of direct methanol fuel cells, J. Power Sources. 180 (2008) 221-226.

[7] M. Huang, Y. Jiang, C. Jin, J. Ren, Z. Zhou, L. Guan, Pt-Cu alloy with high density of surface Pt defects for efficient catalysis of breaking $\mathrm{C}-\mathrm{C}$ bond in ethanol, Electrochim. Acta. 125 (2014) 29-37.

[8] L. Lloyd, Handbook of Industrial Catalysts, Springer, 2011.

[9] T.C.R. Rocha, M. Hävecker, A. Knop-Gericke, R. Schlögl, Promoters in heterogeneous catalysis: The role of Cl on ethylene epoxidation over Ag, J. Catal. 312 (2014) 12-16.

[10] V. Polshettiwar, R.S. Varma, Green chemistry by nano-catalysis, Green Chem. 12 (2010) 743.

[11] B.C. Steele, A. Heinzel, Materials for fuel-cell technologies., Nature. 414 (2001) 34552.

[12] J. Coronado, F. Fresno, M.D. Hernández-Alonso, R. Portela, Design of Advanced Photocatalytic Materials for Energy and Environmental Applications, Springer London, 2013.

[13] H.-J. Freund, G. Pacchioni, Oxide ultra-thin films on metals: new materials for the design of supported metal catalysts., Chem. Soc. Rev. 37 (2008) 2224-42.

[14] Y.-N. Sun, Z.-H. Qin, M. Lewandowski, E. Carrasco, M. Sterrer, S. Shaikhutdinov, et al., Monolayer iron oxide film on platinum promotes low temperature CO oxidation, J. Catal. 266 (2009) 359-368. 
[15] Y. Martynova, M. Soldemo, J. Weissenrieder, S. Sachert, S. Polzin, W. Widdra, et al., CO Oxidation Over Monolayer Manganese Oxide Films on Pt(111), Catal. Letters. 143 (2013) 1108-1115.

[16] Q. Pan, B.H. Liu, M.E. McBriarty, Y. Martynova, I.M.N. Groot, S. Wang, et al., Reactivity of Ultra-Thin ZnO Films Supported by Ag(111) and $\mathrm{Cu}(111)$ : A Comparison to ZnO/Pt(111), Catal. Letters. 144 (2014) 648-655.

[17] S. Prada, L. Giordano, G. Pacchioni, C. Noguera, J. Goniakowski, Properties of Ptsupported iron oxide ultra-thin films: similarity of Hubbard-corrected and hybrid density functional theory description., J. Chem. Phys. 141 (2014) 144702.

[18] D. Shin, S. Sinthika, M. Choi, R. Thapa, N. Park, Ab Initio Study of Thin Oxide-Metal Overlayers as an Inverse Catalytic System for Dioxygen Reduction and Enhanced CO Tolerance, ACS Catal. 4 (2014) 4074-4080.

[19] J. Hagen, Industrial Catalysis: A Practical Approach, Wiley, 2006.

[20] C.S.S.R. Kumar, Surface Science Tools for Nanomaterials Characterization, Springer Berlin Heidelberg, 2015.

[21] M. Che, J.C. Vedrine, Characterization of Solid Materials and Heterogeneous Catalysts: From Structure to Surface Reactivity, Wiley, 2012.

[22] J. Schalwig, P. Kreisl, S. Ahlers, G. Muller, Response mechanism of SiC-based MOS field-effect gas sensors, IEEE Sens. J. 2 (2002) 394-402.

[23] R. Jaaniso, O.K. Tan, Semiconductor gas sensors, Woodhead Publishing Limited, 2013.

[24] K.D. Schierbaum, S. Vaihinger, W. Göpel, H.H. van den Vlekkert, B. Kloeck, N.F. de Rooij, Prototype structure for systematic investigations of thin-film gas sensors, Sensors Actuators B Chem. 1 (1990) 171-175.

[25] A. Samman, S. Gebremariam, L. Rimai, X. Zhang, J. Hangas, G.W. Auner, Siliconcarbide MOS capacitors with laser-ablated Pt gate as combustible gas sensors, Sensors Actuators B Chem. 63 (2000) 91-102.

[26] M. Penza, R. Rossi, M. Alvisi, E. Serra, Metal-modified and vertically aligned carbon nanotube sensors array for landfill gas monitoring applications., Nanotechnology. 21 (2010) 105501.

[27] E. Becker, M. Andersson, M. Eriksson, A.L. Spetz, M. Skoglundh, Study of the Sensing Mechanism Towards Carbon Monoxide of Platinum-Based Field Effect Sensors, IEEE Sens. J. 11 (2011) 1527-1534.

[28] Y. Liu, Y. Lei, Pt-CeO2 nanofibers based high-frequency impedancemetric gas sensor for selective $\mathrm{CO}$ and $\mathrm{C}_{3} \mathrm{H}_{8}$ detection in high-temperature harsh environment, Sensors Actuators B Chem. 188 (2013) 1141-1147. 
[29] M. Wallin, H. Grönbeck, A.L. Spetz, M. Eriksson, M. Skoglundh, Vibrational analysis of $\mathrm{H}_{2}$ and $\mathrm{D}_{2}$ adsorption on Pt/SiO $2 .$, J. Phys. Chem. B. 109 (2005) 9581-8.

[30] M. Andersson, R. Pearce, A. Lloyd Spetz, New generation SiC based field effect transistor gas sensors, Sensors Actuators B Chem. 179 (2013) 95-106.

[31] R. Moos, K. Sahner, M. Fleischer, U. Guth, N. Barsan, U. Weimar, Solid state gas sensor research in Germany - a status report., Sensors (Basel). 9 (2009) 4323-65.

[32] J. Andersson, M. Antonsson, L. Eurenius, E. Olsson, M. Skoglundh, Deactivation of diesel oxidation catalysts: Vehicle- and synthetic aging correlations, Appl. Catal. B Environ. 72 (2007) 71-81.

[33] M. Skoglundh, E. Fridell, Strategies for Enhancing Low-Temperature Activity, Top. Catal. 28 (2004) 79-87.

[34] G. Ertl, H. Knözinger, F. Schüth, J. Weitkamp, Handbook of Heterogeneous Catalysis, 8 Volumes, Wiley, 2008.

[35] K. An, S. Alayoglu, N. Musselwhite, K. Na, G.A. Somorjai, Designed catalysts from Pt nanoparticles supported on macroporous oxides for selective isomerization of nhexane., J. Am. Chem. Soc. 136 (2014) 6830-3.

[36] O.T. Holton, J.W. Stevenson, The Role of Platinum in Proton Exchange Membrane Fuel Cells, Platin. Met. Rev. 57 (2013) 259-271.

[37] M. Ritter, W. Weiss, Fe3O4(111) surface structure determined by LEED crystallography, Surf. Sci. 432 (1999) 81-94.

[38] F. Ringleb, Y. Fujimori, H.-F. Wang, H. Ariga, E. Carrasco, M. Sterrer, et al., Interaction of Water with $\mathrm{FeO}(111) / \mathrm{Pt}(111)$ : Environmental Effects and Influence of Oxygen, J. Phys. Chem. C. 115 (2011) 19328-19335.

[39] J. Knudsen, L.R. Merte, L.C. Grabow, F.M. Eichhorn, S. Porsgaard, H. Zeuthen, et al., Reduction of FeO/Pt(111) thin films by exposure to atomic hydrogen, Surf. Sci. 604 (2010) 11-20.

[40] Y.-N. Sun, L. Giordano, J. Goniakowski, M. Lewandowski, Z.-H. Qin, C. Noguera, et al., The interplay between structure and CO oxidation catalysis on metal-supported ultrathin oxide films., Angew. Chem. Int. Ed. Engl. 49 (2010) 4418-21.

[41] R. Nyholm, J.N. Andersen, U. Johansson, B.N. Jensen, I. Lindau, Beamline I311 at MAX-LAB: a VUV/soft X-ray undulator beamline for high resolution electron spectroscopy, Nucl. Instruments Methods Phys. Res. Sect. A Accel. Spectrometers, Detect. Assoc. Equip. 467-468 (2001) 520-524.

[42] E. Becker, M. Skoglundh, M. Andersson, A.L. Spetz, In situ DRIFT study of hydrogen and $\mathrm{CO}$ adsorption on $\mathrm{Pt} / \mathrm{SiO}_{2}$ model sensors, in: 2007 IEEE Sensors, IEEE, 2007: pp. 1028-1031. 
[43] Y. Joseph, G. Ketteler, C. Kuhrs, W. Ranke, W. Weiss, R. Schlögl, On the preparation and composition of potassium promoted iron oxide model catalyst films, Phys. Chem. Chem. Phys. 3 (2001) 4141-4153.

[44] F. Garbassi, L. Balducci, P. Chiurlo, L. Deiana, A study of surface modification of silica using XPS, DRIFT and NMR, Appl. Surf. Sci. 84 (1995) 145-151.
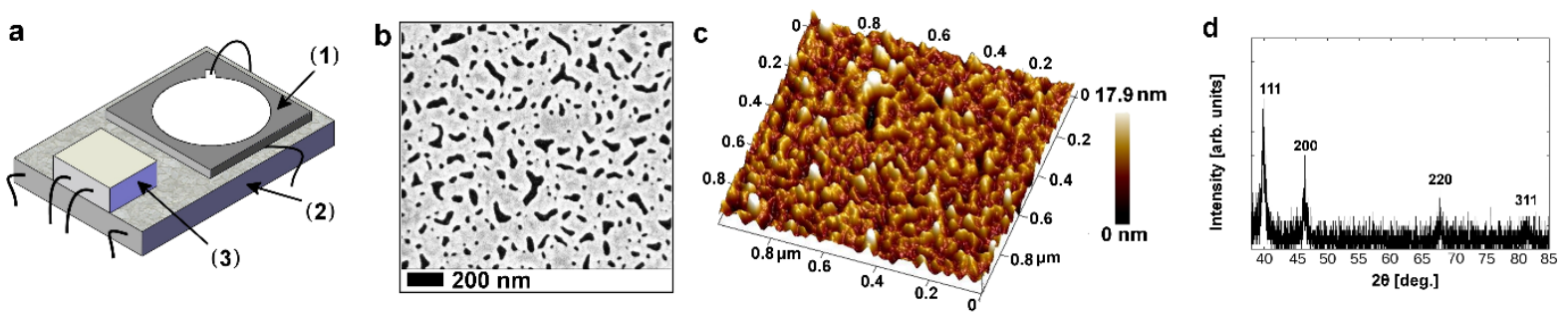

Fig. 1 (a) Schematic illustration of the MOS devices. (1) 4H-SiC chip with circular Pt catalyst layer on top, $4.5 \mathrm{~mm}$ in diameter, and wire bonding to the front side electrical and backside ohmic contacts, (2) heater, (3) Pt-100 temperature sensor. (b) Scanning electron microscopy image of the porous poly-crystalline Pt layer. (c) Atomic force microscopy image of the morphology of Pt surface. (d) X-ray diffraction pattern of a $\mathrm{Pt} / \mathrm{SiO}_{2} / 4 \mathrm{H}-\mathrm{SiC}$ sample showing different diffraction peaks of Pt. 


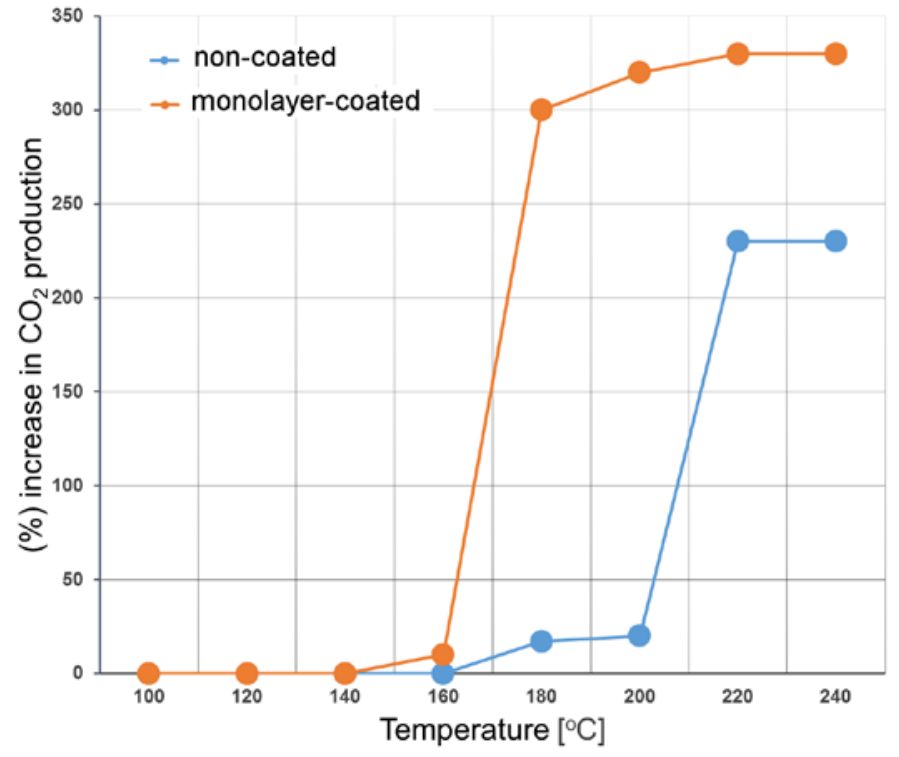

Fig. $2 \mathrm{CO}_{2}$ production over the monolayer-iron-oxide-coated and non-coated MOS model structures at different temperatures. 


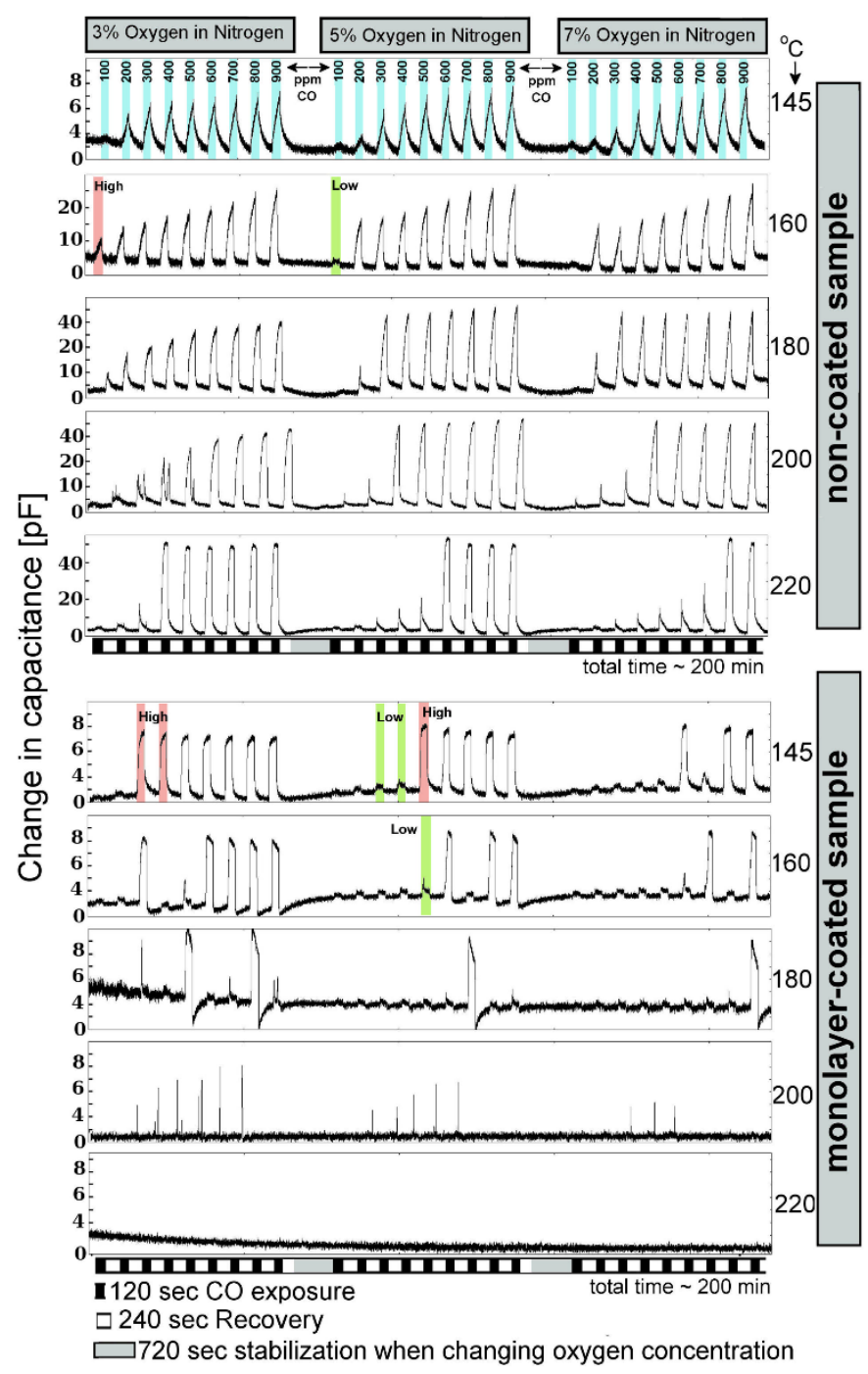

Fig. 3 Responses of non-coated and monolayer-iron-oxide-coated MOS samples to $120 \mathrm{~s}$ carbon monoxide pulses (100 to 900 ppm with 100 ppm steps) separated by 240 s recovery intervals (MOS samples exposed to $\mathrm{O}_{2} / \mathrm{N}_{2}$ carrier gas only), for three different $\mathrm{O}_{2}$ concentrations; 3,5 , and $7 \% \mathrm{O}_{2}$ in $\mathrm{N}_{2}$. The gas exposure details are depicted inside the topmost plot and is similar for all other plots. The output capacitance characteristics are displayed for operation temperatures ranging from 145 to $220^{\circ} \mathrm{C}$. 


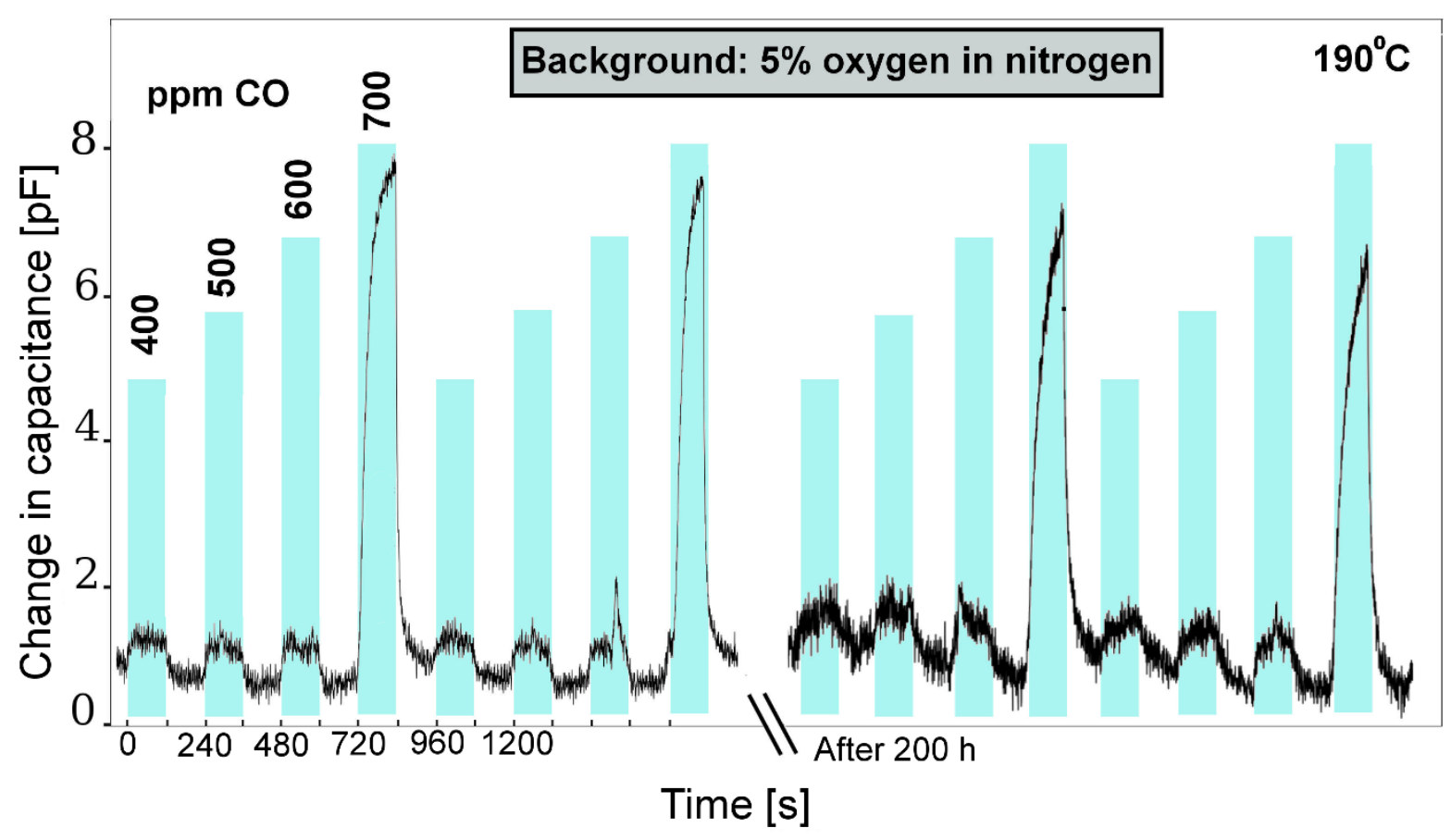

Fig. 4 The stability of the monolayer-iron-oxide-coated MOS capacitor output characteristics to 120 s CO pulses, with 120 s intervals, over 200 hours of constant operation at $190{ }^{\circ} \mathrm{C}$. 

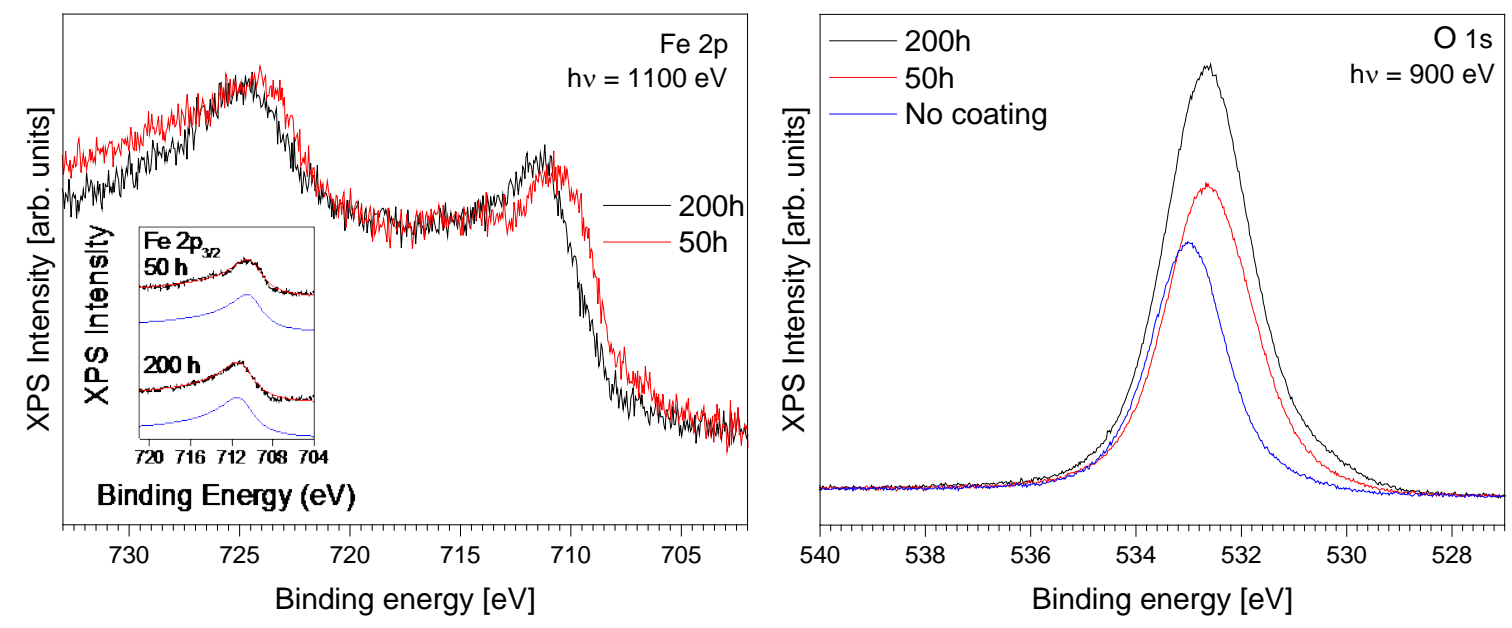

Fig. 5 Photoelectron spectra. (Left) Fe 2p collected at a photon energy of $1100 \mathrm{eV}$ for two samples exposed to 120 s CO pulses for $50 \mathrm{~h}$ and 200 h, respectively (see Fig. 4). (Inset) Peak fits after background subtraction of $\mathrm{Fe} 2 \mathrm{p}_{3 / 2}$ for the $50 \mathrm{~h}$ and $200 \mathrm{~h}$ samples. (Right) $\mathrm{O} 1 \mathrm{~s}$ collected at a photon energy of $900 \mathrm{eV}$ from the same MOS devices as in (left) and a noncoated Pt MOS sample.
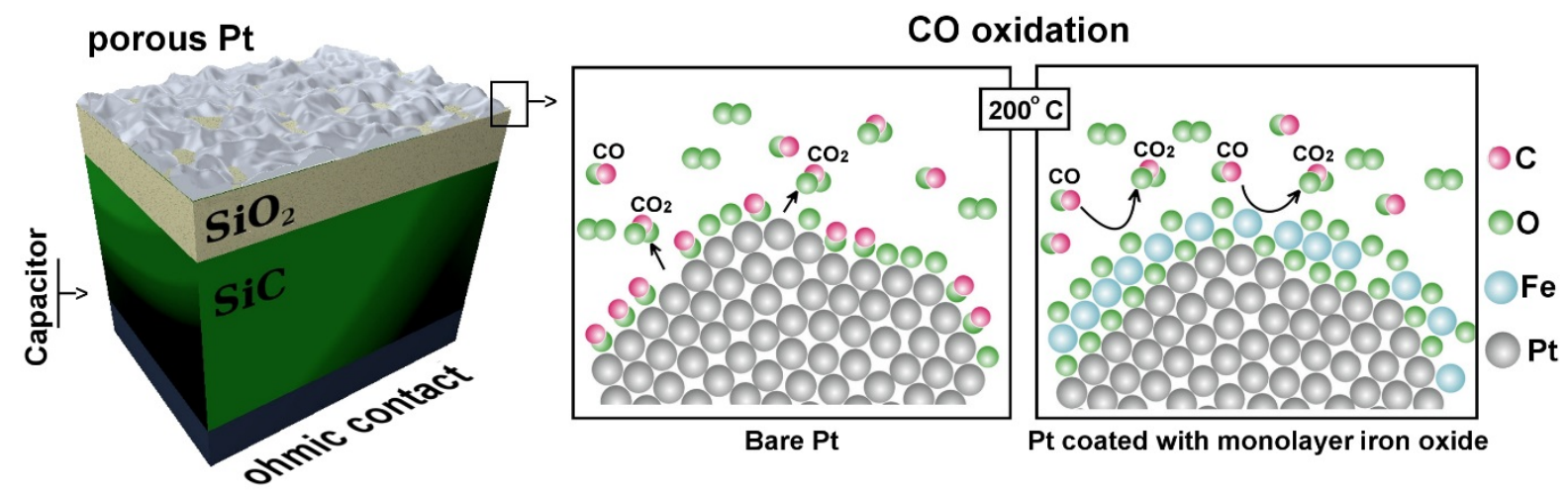

\section{Graphical Abstract}

